\title{
Effect of aluminum nitride buffer layer deposited by molecular beam epitaxy on the growth of aluminum nitride thin films deposited by DC magnetron sputtering technique
}

\author{
B. Riah 1, J. Camus ${ }^{3}$, A. Ayad 1, 2, M. Rammal 3, R. Zernadji ${ }^{3}$, N. Rouag 1, M.A. Djouadi 3,* \\ 1 Laboratoire Microstructures et Défauts dans les Matériaux, Université Frères Mentouri Constantine 1, Route \\ Ain El Bey, Constantine 25017, Algeria ; riah.badis@gmail.com. \\ 2 Département de Pharmacie, Faculté de Médecine, Université Constantine 3, Nouvelle ville Ali Mendjeli, \\ Algeria ; abdelhak.ayad@univ-constantine3.dz. \\ 3 Institut des Matériaux Jean Rouxel IMN UMR 6502, Université de Nantes, 2 rue de La Houssinière BP 32229, \\ 44322 Nantes Cedex France. \\ * Correspondence: riah.badis@gmail.com.
}

\begin{abstract}
This paper reports the effect of silicon substrate orientation and aluminum nitride buffer layer deposited by molecular beam epitaxy on the growth of aluminum nitride thin films deposited by DC magnetron sputtering technique at low temperature. The structural analysis has revealed a strong (0001) fiber texture for both substrates $\mathrm{Si}(100)$ and (111) and a hetero-epitaxial growth on few nanometers AlN buffer layer grown by MBE on Si (111) substrate. SEM images and XRD characterization have shown an enhancement in AIN crystallinity thanks to AIN (MBE) buffer layer. Raman spectroscopy indicated that the AlN film was relaxed when it deposited on $\mathrm{Si}$ (111), in compression on $\mathrm{Si}(100)$ and under tension on AlN buffer layer grown by MBE/Si (111) substrates, respectively. The interface between $\mathrm{Si}(111)$ and AlN grown by MBE is abrupt and well defined; contrary to the interface between AlN deposited using PVD and AlN grown by MBE. Nevertheless, AlN hetero-epitaxial growth was obtained at low temperature $\left(<250^{\circ} \mathrm{C}\right)$.
\end{abstract}

Keywords: Hexagonal AlN, thin films, Direct current magnetron sputtering, Texture, fiber, heteroepitaxial growth.

\section{Introduction}

Aluminum nitride (AlN) thin films can be a promising candidate in optical, mechanical, and electronic applications. It can serve as a semiconductor when doped [1] and also as a passivation layer for semiconductors [2]. Besides, AlN thin films are integrated in surface acoustic wave (SAW) devices, where they insure high frequency ranges, a large electromechanical coupling factor $\left(\boldsymbol{K}_{\mathbf{s}}^{\mathbf{2}}\right)$ and temperature stability of the corresponding device. Nevertheless, the quality of these integrated films has a strong impact on the performance of the SAW devices [3-4]. The film's properties depend not only on the crystal structure of AlN but also, on its preferential orientation [5], c-axis in our case. The films can be composed of multiple crystal orientations scored (002), (100) etc. AlN thin films are grown, using physical vapor deposition (PVD), on several substrates such as silicon (Si) [6], sapphire [7], and indium phosphide (InP) [8]. However, depositing AlN layers on Si simplifies the process and the device structure for a low cost [9].

It is well known that $\mathrm{GaN}$ is one of the most important semiconductors. It is used in blue and ultraviolet light emitting devices as well as high-temperature and high-frequency, high-power electronic devices owing to their excellent properties [10]. Electronic and optoelectronic devices based on GaN deposited on Si (100) and Si (111) substrates are thus highly attractive. GaN films have the potential to be integrated with well-developed Si microelectronic circuits [11]. On the other hand, it is very difficult to 
realize high-quality epitaxial GaN layers on Si. One method for this is to cover the Si substrate by using AlN as a buffer layer, which serves as a substrate for further GaN growth. The AlN has a wide band gap (6.2 eV) III-V compound with high value of thermal conductivity, chemical and thermal stability, refractive index, and breakdown dielectric strength. With these properties, the thermodynamically stable Wurtzite AlN films are not only a good buffer layer materials for GaN growth but also a promising material for applications in other microelectronic and optoelectronic devices [12-14].

In this study, the AIN thin films were deposited using a reactive direct current magnetron sputtering technique (DCMS). AlN films were grown on Si (100), Si (111) substrates and $1 \mathrm{~nm}$ AlN grown by MBE on Si (111) as interlayer. The effect of substrate type on the microstructure and quality of deposited films was studied. It is worth to note that the AlN interlayer grown by MBE has a very good quality with a rocking curve RC-FWHM less than $0.3^{\circ}$ for $150 \mathrm{~nm}$ thick and surface roughness of $1 \AA$ [15]. This interlayer has high electrical resistivity and good thermal conductivity.

The aim of this work is to show that is possible to obtain a hetero-epitaxial growth of AlN films deposited by DCMS method at low temperature on Si (111) by using a few nm AlN buffer layer grown by MBE. The low temperature is very important in order to reduce the thermal expansion problem existing between AlN and GaN. This work is dedicated to the growth of aluminum nitride thin films by PVD techniques at low temperature $\left(<250^{\circ} \mathrm{C}\right)$ and obtain thicknesses up to micron. Such thick and low temperature AlN films will be used in order to ensure thermal management of power electronic devices.

\section{Materials and Methods}

AlN films were deposited using a DCMS technique. An 8 " target was used consisting of 99.99 pure aluminum (Al) water-cooled magnetron cathode. The system was pumped to a base pressure of $10^{-8}$ Torr with a turbo molecular pump before introducing argon (Ar) and nitrogen $\left(\mathrm{N}_{2}\right)$ gases. The sputtering system used to deposit AlN films is a Pinnacle Plus $+5 \mathrm{~kW}{ }^{\circledR}$ DC power supply, with a power of $1800 \mathrm{~W}$. The target was cleaned before deposition using Ar gas discharge followed by a pre-sputtering step using the same conditions as the subsequent film deposition with a shutter shielding the sample in order to remove surface oxidation of the target. The sputtering chamber was evacuated to 2.2 mTorr which is fixed as base pressure. The reactive $\mathrm{N}_{2}$ gas was fixed at $55 \%$. The structural characterization of AlN films was performed by X-ray diffraction (XRD) using a PANanalytical Empyrean ${ }^{\circledR}$ X-ray diffractometer, with $\mathrm{Cu} \mathrm{K} \alpha$ radiation $(\lambda=0.154 \mathrm{~nm})$, voltage and current $(40 \mathrm{kV}-40 \mathrm{~mA})$, respectively. For more details check [16]. In addition, Raman spectroscopy was used in order to obtain the quality of the deposited films and calculate the residual stress which has been developed after deposition. The reflection spectra of the respective molecular structure for the prepared AlN films are identified, studied and presented using Raman spectrometer (Horiba Jobin-Yvon lab-RamT64000 ${ }^{\circledR}$ ) with the green laser of $514 \mathrm{~nm}$ excitation at room temperature. Moreover, scanning electron microscopy (SEM) and the High-Resolution Transmission Electron microscopy (HRTEM) were used in order to thoroughly study the films microstructure, the morphology and the interfaces between the layers. The HRTEM is used only in the case of the AlN deposited on AlN MBE/Si (111).

\section{Results and discussion}

The XRD patterns of the $\theta-2 \theta$ (Bragg-Brentano Geometry) and the $\theta-\theta$ scans of AlN/Si (100), AlN/Si (111) and AlN/AlN MBE (1 nm)/Si (111) are shown in Figure 1 (a) and (b), respectively. The highest intensity of the (0002) reflection at $2 \theta=36.15^{\circ}$ indicates an oriented growth along the $c$-axis perpendicular to substrate in both AlN films depos- 
ited on AlN buffer layer and Si (111). The measure of peak width, the full width at half maximum of the X-ray rocking curve (RC-FWHM) for a determining the quality of the film (Gaussian curve). It can be observed that the FWHM values decreases from $3.2^{\circ}$ to $2.3^{\circ}$ respectively. This result gives an indication that the quality of the AIN films has been improved depending on the Si orientation. On the other hand, the diffraction peak intensity of the (0002) AlN is increased and the RC-FWHM of the AlN film decreased from $2.3^{\circ}$ to $1.2^{\circ}$ using the $\mathrm{AlN}$ (MBE) interlayer, this can indicate that the use of AlN buffer layer enhance the crystalline quality of AIN film and facilitate its deposition. In addition, there is a shift in the peak position of AlN (0002) orientation between the film deposited directly on $\mathrm{Si}(111)$, where $2 \theta=36.09^{\circ}$, and that deposited on AlN MBE (1nm)/Si (111), where $2 \theta=36.15^{\circ}$. This shift is due to the difference in the residual stress developed inside the AlN films.

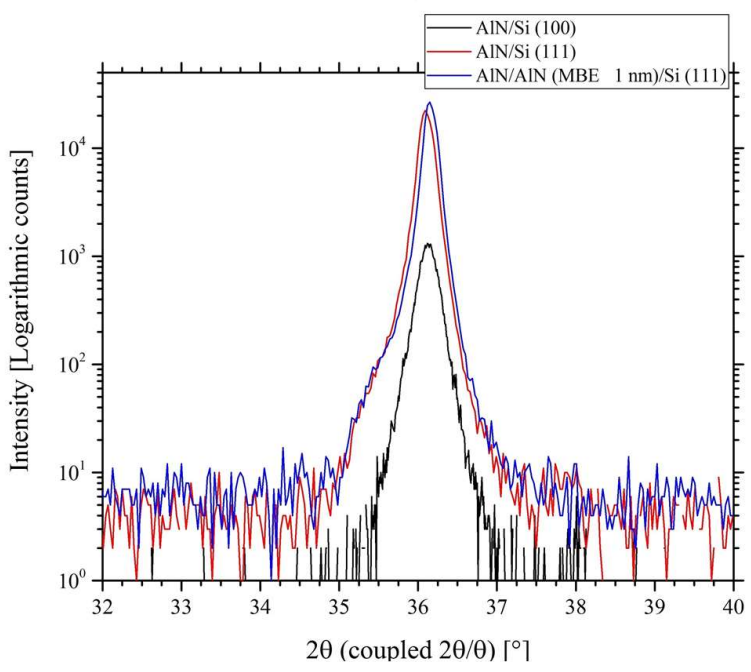

(a)

Figure 1. Diffractogram in the (a) $\theta-2 \theta$ mode and in the (b) $\theta-\theta$ mode showing the AlN peak of (0002) orientation for the samples.

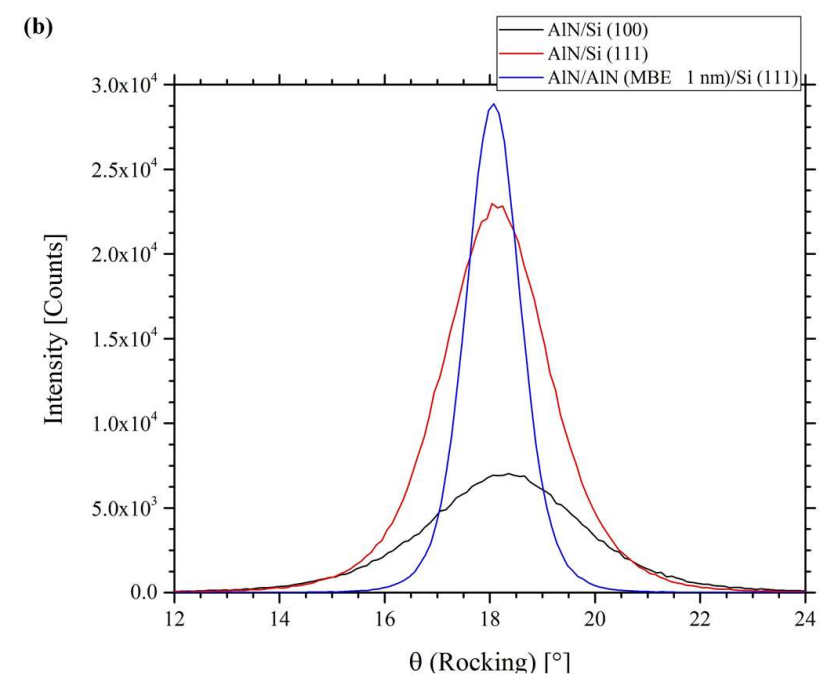

To understand this change, XRD pole figures (0001), \{1011 $\},\{10 \overline{1} 2\}$ and $\{10 \overline{1} 3\}$ of $\mathrm{h}$-AlN were obtained, but only the pole figure $\{10 \overline{1} 1\}$ and $\{10 \overline{1} 2\}$ are used in this study. We characterized, in reflection mode, the incomplete pole figures for $\chi=0$ to $75^{\circ}$ (tilt angle); beyond, the defocus problem becomes too important. The step size is $2.5^{\circ}$ for both angles $\chi$ and $\varphi$ (azimutal angle). The $\{10 \overline{1} 1\}$ pole figures of AlN/Si (100), AlN/Si (111) and AlN/AlN MBE/Si (111) films are shown in Figure 2a, b and c respectively. 

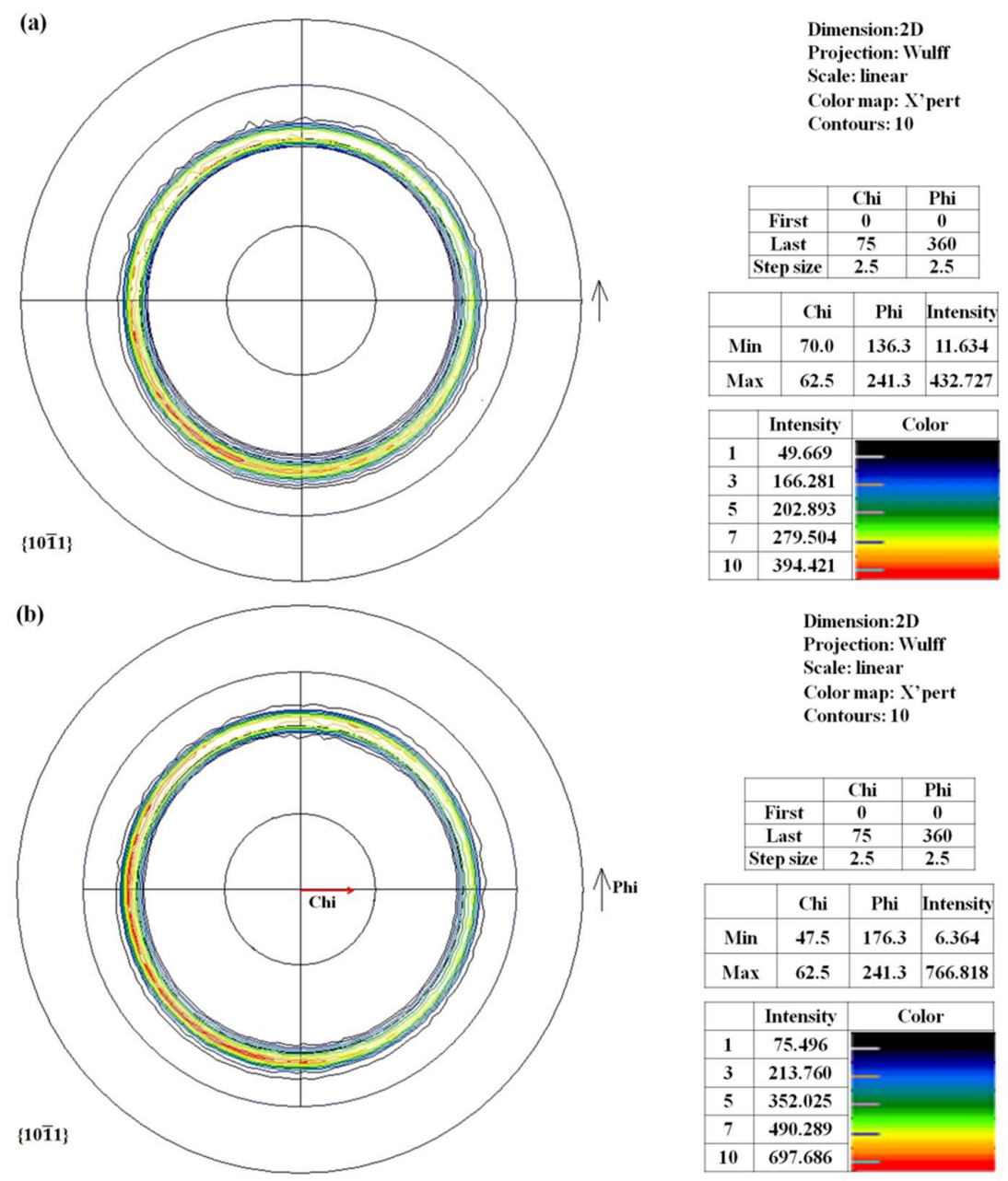

Dimension:2D Projection: Wulff Scale: linear

Color map: X'pert

Contours: 10
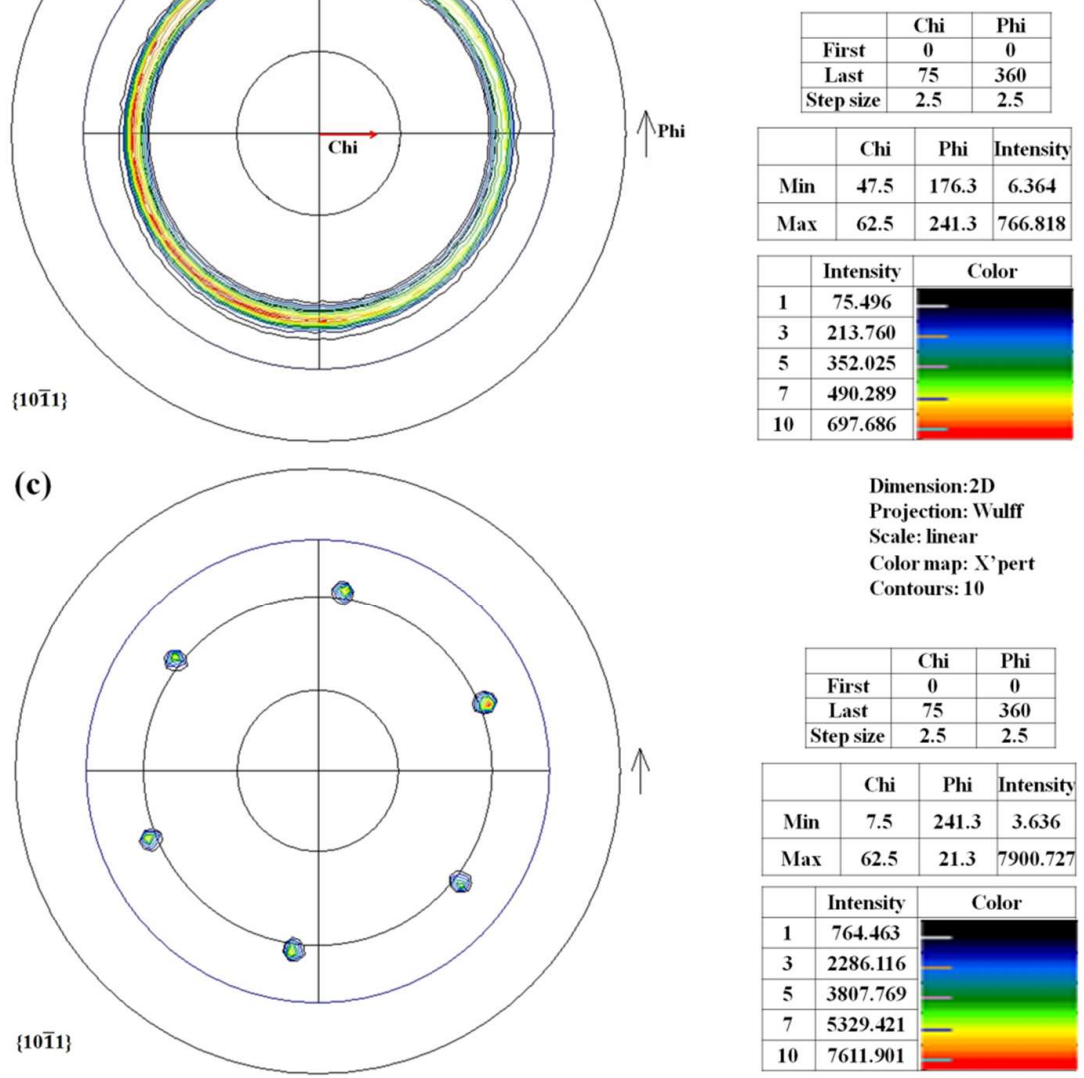

Dimension:2D Projection: Wulff Scale: linear Color map: X'pert Contours: 10

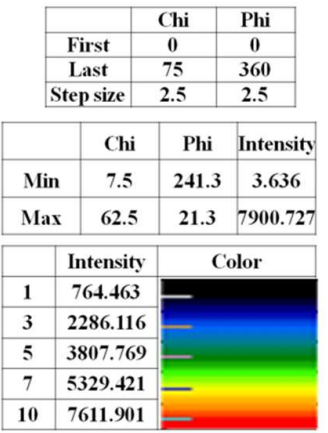

Figure 2: $\{10 \overline{1} 1\}$ pole figures $\left(2 \theta=37.89^{\circ}\right)$ of (a) hexagonal AlN/Si (100) (b) hexagonal AlN/Si (111) and (c) of h-AlN/AlN MBE (1 nm)/Si (111).

For both AlN/Si (100) and AlN/Si (111) samples, the $\{10 \overline{1} 1\}$ pole figures intensity maxima are distributed along a ring located at $\chi=62.5^{\circ}$, demonstrating the presence of a polycrystalline film with (0001) strong fiber-texture, which the grains of the thin film are 
composed of one family of parallel planes at the substrate's surface and having an axis of rotation around the normal of these planes. Moreover, the ring at $\chi=62.5^{\circ}$ caused by the $\{10 \overline{1} 1\}$ facets implies that no preferred in-plane orientation is formed for both cases. On the other hand, the width of the ring is related to the dispersion of (0001) orientation planes. However, in the case of AIN deposited on AIN (MBE) /Si (111), the $\{101 \overline{1}\}$ pole figure shows a maximum intensity with a six-fold symmetry at $\chi=62.5^{\circ}$. It is good to mention that for a $1 \mathrm{~nm}$ thick AlN buffer layer a hetero-epitaxial growth of AlN deposited by DCMS method was obtained. This indicates that the AlN thin film deposited on AlN /Si (111) has not any more a fiber-texture, but has grown epitaxially on the AlN /Si (111) substrate. It can be noticed that the addition of an AIN buffer layer between the deposited AIN PVD and Si (111) substrate allows a hetero-epitaxial growth of the AIN thin films. This can be explained by the lattice mismatch between AlN and Si (100), which is much higher than that with Si (111); thus, the AlN film growth is easier on Si (111). It can be seen that almost 0\% mismatch exist between AIN and AlN buffer layer /Si (111), 19\% between AlN and Si (111) substrate and 43\% in the case of AlN film and Si (100) substrate [16-18].

The morphological and cross sectional characterization of AlN films synthesized at different substrates Si (100), Si (111) and AlN buffer layer/Si (111) were realized using SEM analysis. The cross-section SEM images of deposited films displayed in Fig $3 a, b$ and c. Fig 3a showed an abrupt interface between Si (100) and AlN films deposited by PVD, the AlN layers exhibited an irregular columnar growth with cracks. Fig $3 b$ presented also an abrupt interface between Si (111) and AlN films but it can be seen that there is an inter diffusion between Si substrate and sputtered AlN films with dense columnar structure. However, Fig 3c exhibited the AlN film deposited on AlN buffer layer/Si (111) substrate. This later has an almost regular columnar structure with clear abrupt interface between AlN buffer layer and AIN PVD without defects and cracks, also good film crystallinity. It can be seen that the AlN film deposited on AlN buffer layer/Si (111) has the best columnar structure compared to those deposited on both Si (100) and Si (111) substrates. This confirms the results of XRD analyses shown previously.
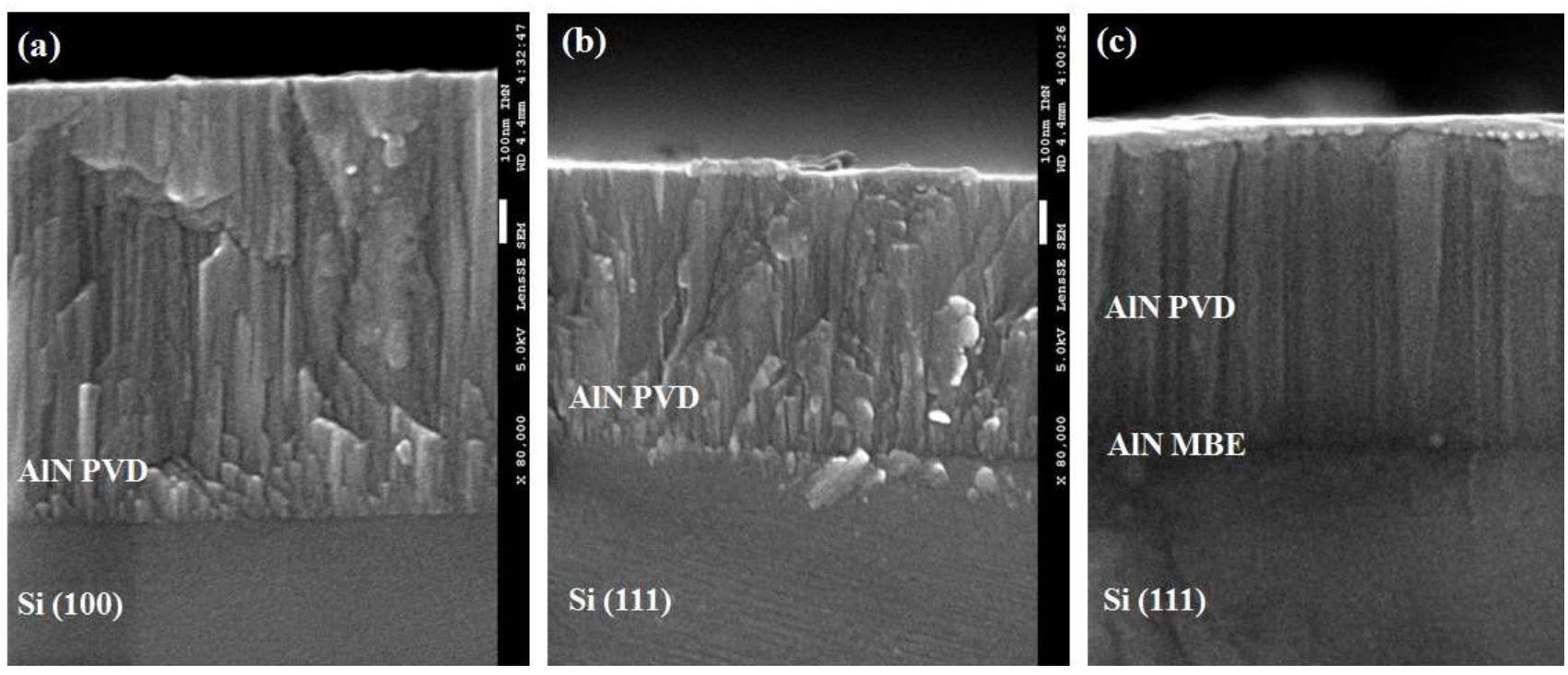

Figure 3. Cross section SEM image for (a) AlN/Si (100), (b) AlN/Si (111) and (c) AlN/AlN MBE/Si (111). 
The samples were also examined by visible Raman spectroscopy at a wavelength of $514 \mathrm{~nm}$ and a full laser power. Raman results provides additional information about the AlN film's quality via the calculation of the High bands $\left(\mathrm{E}_{2}^{\mathrm{H}}\right)$ of the AlN films. Moreover, the $\left(\mathrm{E}_{2}^{\mathrm{H}}\right)$ mode position gives an indication about the residual stress within the film. Figure 4a shows the Raman spectra with AlN bands $A_{1}(T O)$ and $E_{2}^{H}$ for the three samples. One can observe a shift of few nanometers of the $\mathrm{E}_{2}^{\mathrm{H}}$ mode. Moreover, the residual stress developed inside the deposited films was calculated using radius curvature method thanks to Profilometer tests and such data were coupled with Raman shifts. The variation of the stress as function of the $\left(\mathrm{E}_{2}^{\mathrm{H}}\right)$ band shift is represented in Figure $4 \mathrm{~b}$.

It can be seen from Figure 4 that the AlN film deposited on $\mathrm{Si}$ (111) substrate is almost relaxed (Stress $=0.03 \mathrm{GPa}$ ). Nevertheless, for AlN deposited on $\mathrm{Si}(100)$, the stress is equal to -0.37 GPa meaning that the film is in compression. The strain difference in the AlN films deposited on the two types of the substrates can be attributed to the extent of lattice mismatch $[17,19]$. On the other hand, AlN films deposited on AlN buffer layer/Si (111) have shown a stress of $3.23 \mathrm{GPa}$. This result indicates that the film is under tension.

(a)

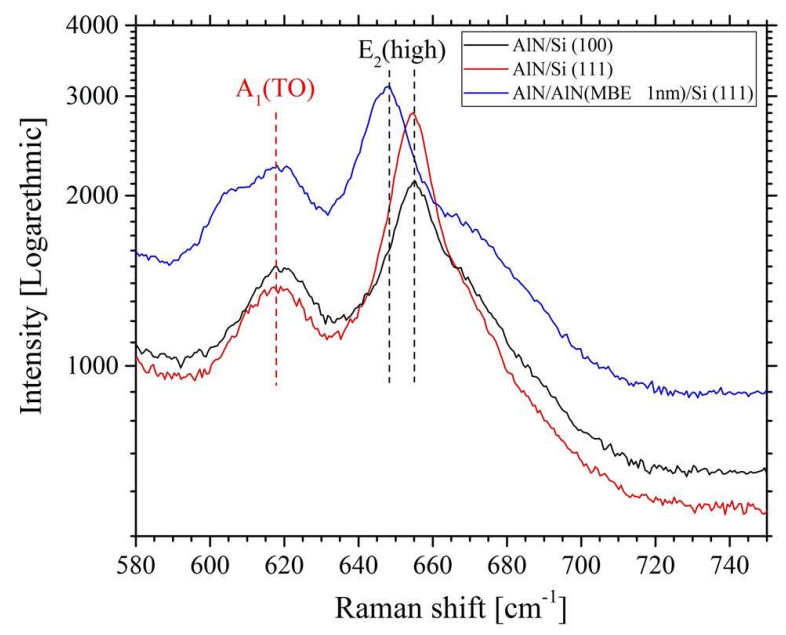

(b)

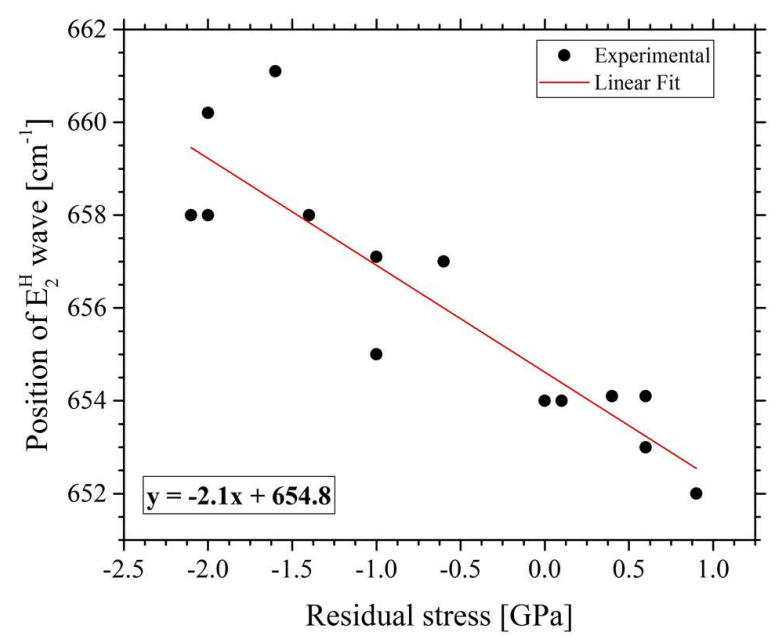

Figure 4. (a) Raman spectra of AlN thin films and (b) Position of the Raman band relative to the AlN (0002) orientation as a function of the residual stress calculated by the method of radius of curvature on Si substrate.

Fig. 5 shows TEM cross section analysis of thick AlN film deposited on few nanometers AlN epitaxial film on Si (111) using DCMS technique. Figure 5 (b) and (c) show a cross section TEM image at low magnification of the AlN layers and Si interface. The contrast differences are observed on $3 \mathrm{~nm}$ from the Si substrate. This difference on the contrast can be representative to the difference between the compositions related to the surface oxidation. It is very important to notice that the AlN thin films and AlN buffer layer are deposited in two different reactors. We have chosen that the surface of the buffer layer was not cleaned to avoid its amorphisation (oxidation). From the TEM images, it appears that the upper AlN exhibits a columnar structure, a very good continuity is observed between both films. The sputtered film is also continuous layer of $3 \mathrm{~nm}$ thick. This layer corresponds to the $1 \mathrm{~nm}$ AlN MBE layer already measured by RHEED technique [20]. Thus, it is obvious that there is an underestimation of the thickness. Moreover, this image confirms that the interface between AlN buffer layer and Si is abrupt. Also, a dense structure is highlighted for the sputtered AlN films followed by column like morphology. 
On the other hand, the interface between the AIN (MBE) buffer layer and AIN deposited by PVD is less abrupt. This is probably due to the presence of defects at the interface. These defects are probably caused by contamination of the buffer layer before AlN sputtering as the two AlN films were deposited in two different reactors. Despite such imperfect interface AIN hetero-epitaxial growth has been reached at low temperature by PVD method.
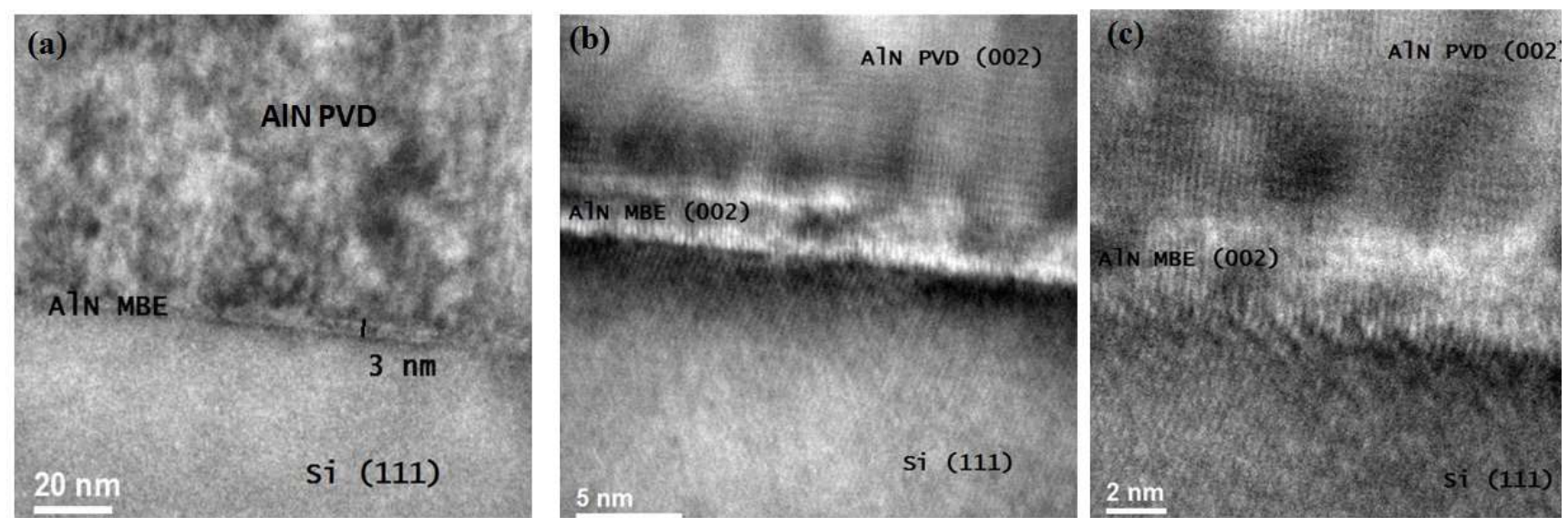

Figure 5. Cross-section HTTEM images at different magnification for the interface between the AIN PVD and the AlN MBE.

\section{Conclusions}

AlN films were grown on Si (111), Si (100) and MBE grown AlN buffer layer on Si (111) substrates with reactive direct magnetron sputtering (DCMS) process at low temperature. The AlN film structures, obtained for all substrates were investigated by XRD, SEM, Raman and TEM analysis and compared. The crystallinity of AlN layers was improved using $3 \mathrm{~nm}$ AlN buffer layer. This layer was found to promote the formation of c-axis oriented AIN layers and suppress the formation of disoriented crystallites on the non-c-axis planes, resulting in the improved crystallinity. Nevertheless, it is thought that only the surface state (oxidation, nitriding, and restructuring) remains an obstacle to a hetero-epitaxial growth of hexagonal AIN directly on Si substrates. The FWHMs of the (0002) X-ray rocking curves were decreased from $3.2^{\circ}$ to $1.2^{\circ}$ using AlN buffer layer. The texture analysis has revealed a strong (0001) fiber texture for both substrates Si (100) and (111) and a hetero-epitaxial growth on $1 \mathrm{~nm}$ AlN buffer layer grown by MBE on Si (111) substrate. It appears that the AlN is respectively relaxed, under compression and under tension when it deposited on Si (111), Si (100) and AlN buffer layer. HRTEM confirmed that the interface between AIN grown by MBE and Si (111) is abrupt. On the other hand, it indicated an unclear interface between the AIN grown by MBE and the AIN deposited by PVD, probably due to the presence of defects. Nevertheless, despite such imperfect interface AIN hetero-epitaxial growth has been reached at low temperature by PVD method.

Author Contributions: Conceptualization, B.R. and M.R.; methodology, B.R, M.R, A.A, N.R.; software, B.R and M.R.; validation, M.A.D.; formal analysis, B.A, M.R and J.C.; investigation, J.C; resources, J.C and M.A.D.; data curation, B.R.; writing-original draft preparation, B.R and M.R.; writing-review and editing, J.C, A.A, M.A.D.; visualization; supervision, M.A.D.; project administration; funding acquisition. All authors have read and agreed to the published version of the manuscript.

Funding: This research received no external funding. 
Institutional Review Board Statement: Not applicable.

Informed Consent Statement: Not applicable.

Data Availability Statement: Data is contained within the article.

Acknowledgments: This work was financially supported in part by the European Union's Horizon 2020 Research and Innovation Program (grant agreement $n^{\circ} 644453$ ). The financial support of DGRSDT is strongly acknowledged.

Conflicts of Interest: The authors declare no conflict of interest.

\section{References}

1. Xiong, J., Guo, P., Guo, F., Sun, X., \& Gu, H. (2014). Room temperature ferromagnetism in Mg-doped AlN semiconductor films. Materials Letters, 117, 276-278. doi: 10.1016/j.matlet.2013.12.018.

2. Aubry, R., Michel, N., Jacquet, J. C., Baczkowski, L., Patard, O., Chartier, E., ... Semmar, N. (2014). S3-P11: Thin-film coatings for improved thermal performances of GaN-based HEMTs. 2014 Lester Eastman Conference on High Performance Devices (LEC). doi: 10.1109/lec.2014.6951566.

3. J.G. Rodríguez-Madrid, G.F. Iriarte, D. Araujo , M.P. Villar , O.A. Williams, W. Müller-Sebert , F. Calle, “Optimization of AlN thin layers on diamond substrates for high frequency SAW resonators", Materials Letters Volume 66, Issue 1, 1 January 2012, Pages 339-342. (SAW).

4. M. Clement, L. Vergara, J. Sangrador, E. Iborra, A. Sanz-Hervas, SAW characteristics of AlN films sputtered on silicon substrates, Ultrasonics 42 (2004) 403-407. (SAW).

5. M. Ishiharaa,U, S.J. Lia , H. Yumotoa , K. Akashib , Y. Idec, Control of preferential orientation of AlN films prepared by the reactive sputtering method, Thin Solid Films 3161998 152-157.

6. Gaurav Shukla, Alika Khare, "Dependence of N2 pressure on the crystal structure and surface quality of AlN thin films deposited via pulsed laser deposition technique at room temperature", Applied Surface Science 255 (2008) $2057-2062$.

7. G.S Sudhir, H Fujii, W.S Wong, C Kisielowski, N Newman, C Dieker, Z Liliental-Weber, M.D Rubin, E.R Weber, “ Pulsed laser deposition of aluminum nitride and gallium nitride thin films", Applied Surface Science, Volumes 127-129, May 1998, Pages 471-476.

8. Pijush Bhattacharya and Dwarka N. Bose, Laser Deposition of AlN Thin Films on InP and GaAs, 1991 Jpn. J. Appl. Phys. 30 L1750.

9. K Ait Aissa, A Achour, O Elmazria, Q Simon, M Elhosni, P Boulet, S Robert and M A Djouadi, “AlN films deposited by de magnetron sputtering and high power impulse magnetron sputtering for SAW applications", J. Phys. D: Appl. Phys. 48 (2015) 145307 (6pp) ; doi:10.1088/0022-3727/48/14/145307.

10. B. Gil, Group III Nitride Semiconductor Compounds: Physics and Application, Clarendon, Oxford, 1998.

11. V. Lebedev, J. Jinschek, U. Kaiser, B. Schroter, W. Richter, J. Kraublich, Appl. Phys. Lett. 76 (2000) 2029.

12. J.X. Zhang, Y.Z. Chen, H. Cheng, A. Uddin, Shu Yuan, K. Pita, T.G. Andersson, “Interface study of AlN grown on Si substrates by radio-frequency magnetron reactive sputtering", Thin Solid Films 471 (2005) 336-341.

13. X.D. Wang, W. Jiang, M.G. Norton, K.W. Hipps, "Morphology and orientation of nanocrystalline AlN thin films", Thin Solid Films 251 (1994) 121.

14. H. Morkoc, Nitride Semiconductors and Devices, Springer, NewYork, 1999, p. 17.

15. J. Camus, Couches de nitrure d'aluminium à basse temperature pour la gestion thermique des composants de puissance. PhD thesis, University of Nantes, (2015).

16. B. Riah, A. Ayad, J. Camus, M. Rammal, F. Boukari, L. Chekour, M.A. Djouadi, N. Rouag, “Textured hexagonal and cubic phases of AlN films deposited on Si (100) by DC magnetron sputtering and high power impulse magnetron sputtering", Thin Solid Films 655 (2018) 34-40.

17. J.X. Zhang, H. Cheng, Y.Z. Chen, A. Uddin, Shu Yuan, S.J. Geng, S. Zhang, Growth of AlN films on Si (100) and Si (111) substrates by reactive magnetron sputtering, Surface \& Coatings Technology 198 (2005) 68- 73.

18. Rammal, M. (2019). Structural and thermal characterization of AlN thin films and their integration in laser devices. PhD thesis, University of Nantes.

19. K. Ait Aissa, A. Achour, J. Camus, L. Le Brizoual, P.-Y. Jouan, M.-A. Djouadi, Comparison of the structural properties and residual stress of AlN films deposited bydc magnetron sputtering and high power impulse magnetron sputtering at different working pressures, Thin Solid Films 550 (2014) 264-267.

20. A.I Nikiforov, B.Z Kanter, O.P Pchelyakov, RHEED investigation of limiting thickness epitaxy during low-temperature Si-MBE on (100) surface, Thin Solid Films, ISSN: 0040-6090, Vol: 336, Issue: 1, Page: 179-182. 